\title{
A maternal Western diet during gestation and lactation modifies offspring's microglial cell density and morphology in the hippocampus and prefrontal cortex in Yucatan minipigs
}

David Val-Laillet ${ }^{1, \#, *}$, Ameni Kanzari ${ }^{1,2, \#}$, Sylvie Guerin ${ }^{1}$, Gwénaëlle Randuineau ${ }^{1}$, Nicolas Coquery $^{1}$

${ }^{1}$ INRAE, INSERM, Univ Rennes, Nutrition Metabolisms and Cancer, NuMeCan, Rennes, St Gilles, France

${ }^{2}$ Université de Tunis El Manar, Faculté des Sciences de Tunis, UR/11ES09 Laboratory of Functional Neurophysiology and Pathology, Tunis, Tunisia.

\# These two authors contributed equally

*Corresponding author: David VAL-LAILLET, INRAE UMR1341 NuMeCan, $35590 \mathrm{St}$ Gilles, France. Email: david.val-laillet@inrae.fr

Short title: Effects of the maternal diet on the offspring's microglia density and morphology in the hippocampus and prefrontal cortex

Highlights

- Western diet during pregnancy and lactation increases microglial cell density in the prefrontal cortex of offspring

- Western diet during pregnancy and lactation predisposes offspring to changes in microglial cells morphology

- Changes in microglial cells were different between the hippocampus and prefrontal cortex

Abstract

Changes in microglial development and morphology can be induced by inflammatory conditions and associated with eating or mood disorders, such as hyperphagia or depression. 
In a previous paper in the minipig model, we showed that maternal Western diet during gestation and lactation decreased hippocampus neurogenesis and food-rewarded cognitive abilities in the progeny. Whether these alterations are concomitant with a central inflammatory process in brain structures involved in learning and memory (hippocampus, HPC), cognitive (prefrontal cortex, PFC), or hedonic (orbitofrontal cortex, OFC) control of food intake is still unknown. In the present study, Yucatan minipigs (Sus scrofa) sows were exposed to two different diets during gestation and lactation (standard, SD N=7 vs. Western diet, WD N=9). Iba1 is a calcium-binding protein specifically expressed in microglia in the brain, which plays an important role in the regulation of the microglia function. Iba1 expression was examined by immunohistochemical analyses in the PFC, OFC and HPC of piglets. The density of microglial cells, as well as their morphology, were assessed in order to have an indirect insight of microglial cell activation state possibly in relationship with neuroinflammation. The density of Iba1-positive cells was higher in the PFC but not in the HPC of WD compared to SD piglets $(p<0.001)$. In the HPC, anterior and dorsolateral PFC, WD piglets had more unipolar cells, contrary to SD that had more multipolar cells $(P<0.0001)$. Opposite effects were observed in the OFC, with SD presenting more unipolar $(P<0.001)$ microglial cells compared to WD. We showed here that maternal diet during pregnancy and lactation had significant effects on morphological changes of microglial cells in the offspring, and that these effects differed between the HPC and PFC, suggesting different response mechanisms to the early nutritional environment.

\section{Abbreviations}

PFC: prefrontal cortex

APFC: anterior prefrontal cortex

DLPFC: dorsolateral prefrontal cortex 
HPC: hippocampus

WD: western diet

SD: standard diet

OFC: orbitofrontal cortex

PND: post-natal day

Keywords: Microglial cell, Prefrontal cortex; Hippocampus, Western diet, Minipig

\section{INTRODUCTION}

The maternal nutritional status plays a major role in the development of the progeny and its susceptibility to diseases. This phenomenon of nutritional programming, or developmental origins of health and diseases $(\mathrm{DOHaD})$, has been initially described by Barker et al. [1,2]. Fetal development depends on the nutrient supply provided by the mother's diet. As a consequence, any alteration of the mother's metabolism may also predispose the progeny to metabolic anomalies. Maternal high-fat diet consumption during gestation and/or lactation may increase the susceptibility of the progeny to declare metabolic or neuropsychiatric disorders in adulthood. Many studies have described the behavioral and brain changes/plasticity induced by maternal obesity. Notably, nonhuman primate offspring of mothers fed a high-fat and high-sugar diet during pregnancy and lactation show increased anxiety behavior [3].

Evidences from both epidemiological studies and animal models demonstrated that high-fat diet consumption during gestation/lactation may promote inflammatory mechanisms in the offspring, because high-fat and high-sugar diets are associated with exaggerated systemic levels of inflammatory mediators [4,5]. Also, the consumption of unbalanced diets can modulate neurophysiological processes, microglial phenotypes, and cause post-natal inflammation [6,7]. Findings from rodent and cell culture models showed that 
neuroinflammation, for which microglial cells are a major actor, is a main cause of cellular and molecular changes in the brain $[8,9]$. In order to study microglial cells, Iba1 represents an excellent antigen to examine morphological changes in microglia, and has been used extensively for this purpose [10-12].

Excess expression of pro-inflammatory cytokines in the brain is related to emotional and cognitive perturbation [13]. Therefore, non-healthy nutrition can have detrimental effects such as reduced cortical neuroplasticity in the human, notably in brain regions involved in emotional and cognitive functions [14]. However, little is known about a differential effect of neuroinflammation on these regions. Are the different brain regions homogenously impacted or are there more sensitive brain areas that could be preferentially affected by neuroinflammation, with specific behavioral consequences?

The prefrontal cortex (PFC) and hippocampus (HPC) are the most frequently studied brain areas to investigate the effects of stress, including inadequate nutritional environments, on neuroinflammation and microglia [15]. In humans, the PFC is strongly involved in food choices, decision-making, flexible cognitive functions, and the regulation of attention and emotions [16-20], whereas HPC is a brain structure essential to memory and learning [21]. In the PFC of mice, it has been shown that microglial cell activation could initiate apoptotic processes and the activation of caspases through the release of a variety of pro-inflammatory and neurotoxic factors [22]. The PFC is essential for the translation of emotional information into behavioral and physiological responses to stress [23,24], and it participates in neural mechanisms underlying the adaptation to stress and pathology [23]. Interestingly, a nutritional n-3 polyunsaturated fatty acids (PUFA) deficiency induced a chronic stress state reflected by a hyperactivity of the hypothalamic-pituitary-adrenal (HPA) axis in mice. This hyperactivity in turn resulted in neuronal atrophy in the dorsolateral (dl)- and dorsomedial (dm)-PFC, and in subsequent mood-related behavioral alterations, similarly to a chronic social defeat stress 
[25]. Another study showed that intrauterine growth restriction, which is known to be associated with neuroinflammation [26], increased impulsive behavior and was associated with altered dopamine transmission in both medial prefrontal and orbitofrontal cortex in rats [27]. Neuroinflammation can increase the number of Iba1-positive microglial cells in PFC of mice susceptible to anhedonia [28-30]. The metabolic profile of the mother has a significant impact on developing offspring. Neuroinflammation, a factor associated with maternal obesity [31], may induce PFC dysfunction in the offspring, and this dysfunction may be associated with an increased risk of mental disorders, including anxiety and depression as demonstrated in nonhuman primates and rats [3,32], as well as attention deficit hyperactivity [33] and autism spectrum disorders [34] as shown in humans. These results demonstrate that early exposure to high-fat diet can directly induce inflammation in the PFC. Conversely, a perinatal maternal obesity can also result in adult HPC insulin resistance in a mouse model, with decreased HPC signaling and impaired expression of markers of neurogenesis (doublecortin), synaptic plasticity (FoxO1, pSynapsin) and function in the offspring [35]. Perinatal exposure to gestational diabetes mellitus in rats has also been described to promote an increased number of ameboid microglial cells in the HPC of the progeny [36].

Investigating the microglia status in the pig model, of which the assets in nutritional and neurosciences studies have been extensively praised [37,38], might help describing the relationships between perinatal high-fat/sugar diet, PFC- and HPC-related cognitive performances, as well as neuroinflammation in the progeny. In addition to its numerous similarities with the human in terms of general anatomy and physiology, especially at the gut and brain levels, the pig has been extensively used as a model for perinatal nutrition and metabolism [39-41]. Our group also has a 10-year expertise in the use of brain imaging in the (mini)pig model for nutrition studies [38] and was the first to describe brain anomalies in obese minipigs similar to those described in obese human patients [42]. The fact that the pig 
has a digestive system very close to that of humans [38] as well as a brain quite similar to that of nonhuman primates [43] justifies its use as a preclinical animal model of predilection and facilitates the translation of results to the human.

In a recent study performed in our lab in the Yucatan minipig model [44,45], we showed that maternal Western diet (WD) during gestation and lactation altered the hippocampal development and neurogenesis, while promoting behavioral changes in the progeny, in terms of cognition and/or motivation for sweet and fatty food rewards. In the young age, piglets exposed to WD during the perinatal period also showed higher plasma cholesterol and free fatty acids concentrations, as well as a decreased gut microbiota fermentation activity [44]. At the adult age, WD normal-weight pigs demonstrated a lower glucose tolerance and a tendency to a higher incremental area under curve of insulin after intravenous glucose injection [45]. We can hypothesize that an inflammation process at the level of the HPC and/or PFC in the young age, indirectly assessed with microglial cell density and morphology, might have provoked the alteration of the neuroplasticity and behavior observed. We also aimed here at investigating whether the changes in microglial cell density and morphology might be different between the ventral HPC and the different subdivisions of the PFC, such as the orbito-frontal cortex (OFC), the anterior prefrontal cortex (A-PFC) and the dorsolateral prefrontal cortex (DL-PFC) that are also involved in different brain functions. Different profiles in terms of microglial status between these brain regions might provide potential explanations for the behavioral changes previously observed in the progeny further WD perinatal exposure $[44,45]$.

\section{MATERIALS \& METHODS}

\subsection{Animals and housing}

This experiment was conducted at INRAE (Institut National de Recherche pour l'Agriculture, l'Alimentation et l'Environnement) of St Gilles, France, in accordance with the 
current ethical standards of the European Community (Directive 2010/63/EU), Agreement No. A35-622 and Authorization No. 35-88. The Regional Ethics Committee in Animal Experiments of Brittany has validated and approved the entire procedure described in this paper (project $\left.\mathrm{N}^{\circ} 01299.01\right)$.

Seventeen Yucatan minipig sows from the experimental breeding station of INRAE St Gilles (France) were used in this study as already described [44]. Sows and their piglets were weighed once a week throughout the whole experiment. Complete details about animals housing conditions, feeding plan, dietary treatment group and dietary treatment effect on body weight of sows and piglets are fully accessible in Val-Laillet et al. [44]. Briefly, from 4 weeks after fecundation, gestation was confirmed by ultrasonography, and the Yucatan sows received either a standard (SD) or high-energy high-fat-fructose western diet (WD) feed until their offspring's weaning at post-natal day (PND) 25 [44]. From weaning onwards to euthanasia at PND 90, piglets from both groups (68 animals in total) were subjected to the same standard diet for piglets (SDP), and only 16 piglets were used in the present immunohistological study. The composition of all experimental diets is presented in Table $\mathbf{1}$.

\subsection{Immunohistochemistry and microglial cell density determination}

Euthanasia, brain sampling and immunohistochemistry procedures are described in Val-Laillet et al. [44]. The number of immunoreactive cells (Iba1 positive) was evaluated in the right hemisphere. In the PFC, two brain sections, respectively at $4.5 \mathrm{~mm}$ and $9.0 \mathrm{~mm}$ from the most anterior part of the brain were used (Fig. 1A) and four regions of interest were selected, including the HPC and the three different subzones of the PFC identified in our digital pig brain atlas [46]: anterior (APFC) and dorsolateral prefrontal cortex (DLPFC), as well as orbitofrontal cortex (OFC). The first section corresponded to the APFC, in which cell density was determined by averaging cell density within 5 squares. The second section corresponded to the DLPFC, in which cell density was determined by averaging cell density 
within 4 squares, and the OFC, in which cell counting was performed in only 1 square, because of the smaller size of this brain region (Fig. 1B, in red). The microglial cell density in the PFC was determined by averaging the cell density within these three brain regions. In the HPC, Iba1-positive cell density was determined along 11 ventral hippocampal brain sections, in which counting was performed within three squares per section located in the hilus (Fig. 1C). After averaging within each brain section, the mean Iba1-positive cell density was calculated for the whole 11 brain sections. For all counting, we used a square of $1.6 \mathrm{~mm}^{2}$ surface for cell density computation.

Immunohistochemistry and image acquisition were performed as followed. After three rinses in phosphate buffered saline (PBS), $150 \mu \mathrm{L}$ of blocking buffer (PBS solution, Gibco by Life Technologies; $10 \%$ horse serum, Sigma; $0.3 \%$ Triton, Sigma) was deposited on the region of interest and stored during $1 \mathrm{~h}$ at ambient temperature in a humidity chamber. Blocking buffer was removed and $150 \mu \mathrm{L}$ of primary antibody (Iba1: Anti-Iba1 Rabbit 1/200, Wako, Sobioda) was deposited, and slides were stored at $4^{\circ} \mathrm{C}$ during at least $16 \mathrm{~h}$. After three rinses in PBS, $150 \mu \mathrm{L}$ of secondary antibody $\left(\mathrm{Cy}^{\mathrm{TM}} 3\right.$-conjugated AffiniPure Donkey AntiRabbit IgG (H+L) 1/500, Jackson ImmunoResearch Laboratories) was deposited on the slices and incubated during $2 \mathrm{~h}$ at ambient temperature in a humidity chamber. After three rinses in PBS, slides were mounted with Fluoroshield Mounting Medium with DAPI (2 drops per slide, Abcam) and stored at ambient temperature until observation. Sections were examined under a fluorescent microscope (Nikon Eclipse 80i, Nikon), digitized and large field mosaics (with X10 objective) were performed with micro-manager (ImageJ plugin).

\subsection{Microglial cells morphology}

Microglial cell morphology was determined knowing that morphological changes might be indirectly related to functional activation of the microglial cells in the context of 
diseases $[47,48]$. We thus determined microglial cell morphology by counting the cellular processes branching off the soma, given that a brain section thickness of $30 \mu \mathrm{m}$ can be used to perform a sufficient estimation of this parameter. Distal arborization is typical of "ramified" microglia, and determination of the number of extensions is an indicator of the arborization complexity. This term of "ramified" microglia has been associated with "resting" microglia, suggesting a potential link between morphology and function [28-30].

To determine these morphological changes or states, one square per subregion of the PFC, i.e. APFC, DLPFC and OFC, and three squares in one hippocampal brain section were selected to describe the morphology of the Iba1-positive microglial cells (Fig. 1B). Each square contained at least 30 microglial cells. The different morphological types of microglial cells were determined on the basis of their polarity number [49] (one polarity: uni-polar; two polarities: bi-polar; more than two: multi-polar; Fig. 2). Some Iba1-positive cells could not be clearly attributed to one cellular type and were consequently not included in the analysis. Note that there were no statistical differences between groups for this non-attributed type of microglial cells (data not shown).

\subsection{Data Analysis}

Data are expressed as mean \pm standard error of the mean (SEM). ANOVA tests were performed with SPSS to determine statistical significance. A p-value $<0.05$ was considered significant.

\section{RESULTS}

\subsection{WD maternal exposure alters microglial cell density in PFC but not in HPC}

The number of Iba1+ cells as seen in Fig. 3A was significantly increased in the PFC of WD piglets compared to SD piglets $\left(\mathrm{F}_{(1,14)}=23.32 ; p=0.0003\right.$, Fig. 3B $)$. However, the number of Iba1+ cells was similar between dietary treatments in the HPC (Fig. 3C). 
3.2 WD maternal exposure differently modifies microglial cell density in three PFC subregions

In the APFC, WD piglets presented a higher number of unipolar cells compared to SD $\left(\mathrm{F}_{(1,14)}=61.28 ; p<0.0001\right)$, whereas SD piglets presented a higher number of multipolar cells compared to WD $\left(\mathrm{F}_{(1,14)}=139.61 ; p<0.0001\right)$. In the DLPFC, WD piglets presented a higher number of unipolar cells compared to $\mathrm{SD}\left(\mathrm{F}_{(1,14)}=44.18 ; p<0.0001\right)$, whereas SD piglets presented a higher number of multipolar cells compared to $\mathrm{WD}\left(\mathrm{F}_{(1,14)}=378.00 ; p<0.0001\right)$. $\mathrm{SD}$ also presented a higher number of bipolar cells compared to $\mathrm{WD}\left(\mathrm{F}_{(1,14)}=12.60 ; p<0.01\right)$. In the OFC, WD piglets presented a higher number of multi-polar cells compared to $\mathrm{SD}\left(\mathrm{F}_{(1}\right.$, 14)=272.46; $p<0.0001$ ), whereas SD piglets presented a higher number of unipolar cells compared to $\mathrm{WD}\left(\mathrm{F}_{(1,14)}=18.64 ; p<0.001\right)($ Fig. 4A).

3.3 WD maternal exposure modifies microglial cell morphology in the HPC toward an increased unipolar category

In the HPC, WD piglets had much more unipolar microglial cells compared to $\mathrm{SD}\left(\mathrm{F}_{(1}\right.$, 13) $=34.59 ; p<0.0001)$, whereas SD piglets had much more microglial multipolar cells compared to $\mathrm{WD}\left(\mathrm{F}_{(1,13)}=31.57 ; p<0.0001\right)$. No significant difference was observed between groups for the microglial bipolar cells (Fig. 4B).

\subsection{Body weight evolution and correlations with Iba1}

Dietary treatment did neither affect average piglet body weight at birth $(\mathrm{SD}, 0.78 \pm 0.05$ vs. WD, $0.81 \pm 0.04 \mathrm{~kg}, p>0.05)$ and weaning (SD, 9.56 $\pm 0.55 v s . \mathrm{WD}, 8.96 \pm 0.57 \mathrm{~kg}, p>0.05)$, nor body weight development from birth up to euthanasia (PND 90). There was no significant correlation ( $p>0,10$ for all) between body weight at PND 90 and Iba1 density in the HPC $\left(\mathrm{R}^{2}=0.287\right), \operatorname{APFC}\left(\mathrm{R}^{2}=0.074\right), \operatorname{DLPFC}\left(\mathrm{R}^{2}=0.009\right)$, and $\mathrm{OFC}\left(\mathrm{R}^{2}=0.018\right)$.

\section{DISCUSSION}


We showed in this study that maternal diet during pregnancy and lactation had significant effects on cell density and morphological changes of microglial cells in the offspring. The morphological changes observed in microglia were perhaps related to the inflammatory status of the CNS, which might have impacted brain functions as well as physiological and behavioral responses. Interestingly, we did detect differences in microglial cell density and morphology between the brain regions studied, suggesting that some brain regions are more sensitive to diet-based neuroinflammation.

\subsection{Impact of the maternal diet on microglia density}

The first results observed in the WD piglets were the increase in the microglial density at the level of the PFC and its sub-regions (APFC, DLPFC, OFC) but not in the ventral part of HPC. This suggests that changes in microglial cell density can differ between specific brain regions and therefore modulate specific neurocognitive functions [50]. In our piglets, a western diet effect in the density of microglia cells in the PFC could be attributed to several factors. First, the significant increase in microglial cell density at the level of PFC in WD piglets might be a response to the perinatal exposure to the diet that might have promoted inflammation [5,7]. This early inflammation might have increased the number of microglia cells in the PFC, which is the site of various cognitive functions encompassing flexible adaptation to changing environments [51], as well as cognitive inhibitory control over food intake and other executive functions. It is interesting to note that this increased microglial cell density was detected after weaning when all animals were fed a standard diet. This demonstrates that the impact of maternal WD might condition the microglial cells development and brain plasticity of the progeny long after the in utero and suckling periods. Conversely, we could not detect any difference in microglial cell density in the ventral part of the HPC. However, an induction of inflammation by the vulnerability to stress can increase the density and alter the functions of the microglia in the ventral HPC of rats [52]. Lesion of 
dorsal, but not ventral, HPC by injection of ibotenic acid causes severe deficits in spatial learning in a water maze [53]. Consequently, the absence of difference in microglial cell density in the ventral HPC does not preclude the effects that might have been observed in the dorsal HPC, which highlights the necessity to explore both sub-regions for further studies. Additionally, the microglial cell density in the HPC was counted as nearly as six times higher than in the PFC. We thus hypothesize that with a higher microglial cell density in normal condition, the HPC might be less sensitive than the PFC to a local increase of cell number. However, as described latter in the discussion, the microglial cell morphology profiles were similar between the A-PFC, the DL-PFC and the HPC, suggesting that the process leading to the WD-based changes might be similar between these brain regions.

\subsection{Impact of the maternal diet on microglia morphology}

The increased microglial cell density was associated with morphological changes characterized by a decreased number of microglial extensions at the level of the HPC, APFC and the DLPFC in WD piglets. This effect was not found in the OFC though. Overall, even if a dedicated analysis aimed at validating microglial cell activation is needed in order to confirm neuroinflammation, the decreased number of microglial extensions suggests such a phenomenon in the HPC, APFC and DLPFC of WD piglets. Inversely, we could detect a higher number of microglial cells with multiple extensions in the OFC of WD piglets, suggesting lower neuroinflammation. The prefrontal cortex has been classically defined and delineated by anatomical criteria such as cytoarchitectonic characteristics (granular vs. agranular characteristics) [54], and efferent and/or afferent projections [55]. In the pig model, the prefrontal cortex is generally divided into three topologically different regions: the APFC, DLPFC and OFC. Some evidences suggest that, in the prefrontal cortex, different subregions underlie distinct cognitive functions [56,57]. In particular, the dorsal and anterior regions of PFC are involved in working memory and attentional tasks, while more orbital regions, such 
as the $\mathrm{OFC}$, are related to visceral functions and rewarding treatments, especially in relation to food [55]. Neuroinflammation can impact differently the brain regions depending on their architecture and function. For example, chronic stress induces dendritic loss in medial PFC and HPC [58,59], but dendritic growth in OFC and basolateral amygdala [60]. Thus, morphological changes as well as effector functions of activated microglia following stimuli (e.g. inflammation) can be specific to the different regions of the brain [61], and probably also specific to their processes.

\subsection{Malnutrition-induced brain anomalies and inflammation are independent to weight} gain

Even though our WD piglets did not gain more weight than SD animals, their physiological and behavioral responses were altered [44]. This was concomitant with different effects on microglial cells according to the brain structure considered. However, changes in microglial cells were not correlated with body weight. The increased microglia density (in APFC, DLPFC, and OFC) and decreased number of microglial extensions (in HPC, APFC, DLPFC) are interpreted as indicators of neuroinflammation. Previous work demonstrated in mice that WD exposure, even in the absence of obesity, could induce prefrontal cortex inflammation as well as cognitive and motor deficits [62]. Gomes et al. [63] also demonstrated that the chronic consumption of high-refined carbohydrate diet, without any significant increase in body weight, activated microglial cells in the HPC and PFC and facilitated anxiety-like behavior in mice through neuroinflammation. We also stated in the introduction that intrauterine growth restriction, which is usually associated with neuroinflammation, could alter the dopamine transmission in both medial prefrontal and orbitofrontal cortex in rats [27].

In the context of our project, over the 68 piglets that were born from our SD and WD sows, only 16 were used for immunohistological investigation in the young age. The 
remaining piglets were kept alive and fed a standard diet until the adult age for subsequent behavioral and brain explorations [45]. In this follow-up study, we demonstrated that normalweight adult WD minipigs expressed a higher stress level, lower performance in the alley maze test, as well as lower dopamine transporter binding in hippocampal structures, associated with lower basal brain activity in the prefrontal cortex compared with normalweight adult SD minipigs [45]. We do not know yet whether these anomalies observed in normal-weight adults are connected with the microglia changes detected in the young age. But it seems that neuroinflammation, especially in prefrontal and hippocampal structures, is not a specificity of obesity or overweight, and can be induced by many inadequate nutritional environments, even in normal-weight individuals.

\subsection{General conclusion}

In conclusion, this study demonstrates that perinatal exposure to a WD diet is accompanied with morphological and cell number changes in microglia in the PFC/HPC, via the potential onset of central inflammation. However, all these brain regions did not respond similarly, the HPC showing no difference in WD-induced microglial cells density, and the OFC showing a specific profile of microglial cell morphology, which suggests less neuroinflammation compared to the other PFC brain regions and the HPC. These results open the way to further studies and paradigms investigating the perinatal WD diet effects on brain and behavior, as well as their contribution to abnormal neurological development in younglings. Future studies should be aimed at identifying why particular brain regions are more sensitive to the nutritional environment than others, which specific factors favor the onset of neuroinflammation, and how this particular adaptive process can modulate brain functions and behavior.

\section{Authors' contribution}


Experimental design: NC and DVL. Technical development: NC, SG, and GR. Performing the experiments: AK, SG, and GR. Data analysis: AK and NC. Manuscript writing: DVL, AK, and NC. Manuscript revising: all co-authors.

\section{Acknowledgements and Fundings}

This study was funded by the INRAE DID'IT (Diet Impact and Determinants: Interactions and Transitions) Metaprogramme in the context of the SweetLip-Kid project coordinated by Sophie Nicklaus. Ameni Kanzari received a grant from the University of Tunis for her internship at INRAE. The authors gratefully acknowledge the efforts and cooperation of the technical staff at INRAE St Gilles, UEPR (Unité Expérimentale Porcs de Rennes): Armel Delalande, Mickaël Génissel, Julien Georges, Régis Janvier, Alain Chauvin, Francis Le Gouevec and Vincent Piedvache.

\section{REFERENCES}

[1] D.J.P. Barker, C. Osmond, P.D. Winter, B. Margetts, S.J. Simmonds, WEIGHT IN INFANCY AND DEATH FROM ISCHAEMIC HEART DISEASE, The Lancet. 334 (1989) 577-580. https://doi.org/10.1016/S0140-6736(89)90710-1.

[2] D. Barker, The malnourished baby and infant: relationship with type 2 diabetes, 60 (2001).

[3] E.L. Sullivan, B. Grayson, D. Takahashi, N. Robertson, A. Maier, C.L. Bethea, M.S. Smith, K. Coleman, K.L. Grove, Chronic Consumption of a High-Fat Diet during Pregnancy Causes Perturbations in the Serotonergic System and Increased Anxiety-Like Behavior in Nonhuman Primate Offspring, J. Neurosci. 30 (2010) 3826-3830. https://doi.org/10.1523/JNEUROSCI.5560-09.2010.

[4] J.L. Bolton, S.D. Bilbo, Developmental programming of brain and behavior by perinatal diet: focus on inflammatory mechanisms, Dialogues Clin. Neurosci. 16 (2014) 307320.

[5] E.L. Sullivan, L. Nousen, K. Chamlou, Maternal High Fat Diet Consumption during the Perinatal Period Programs Offspring Behavior, Physiol. Behav. 123 (2014). https://doi.org/10.1016/j.physbeh.2012.07.014.

[6] R.B. Canani, M.D. Costanzo, L. Leone, G. Bedogni, P. Brambilla, S. Cianfarani, V. Nobili, A. Pietrobelli, C. Agostoni, Epigenetic mechanisms elicited by nutrition in early life, Nutr. Res. Rev. 24 (2011) 198-205. https://doi.org/10.1017/S0954422411000102.

[7] A.-L. Dinel, C. Joffre, P. Le Ruyet, S. Layé, O53: Impact des lipides laitiers pendant la période périnatale sur l'inflammation et la neurogénèse, Nutr. Clin. Métabolisme. 28 (2014) S57-S58. https://doi.org/10.1016/S0985-0562(14)70629-6.

[8] B. Liu, J.-S. Hong, Role of Microglia in Inflammation-Mediated Neurodegenerative 
Diseases: Mechanisms and Strategies for Therapeutic Intervention, J. Pharmacol. Exp. Ther. 304 (2003) 1-7. https://doi.org/10.1124/jpet.102.035048.

[9] T. Wyss-Coray, L. Mucke, Inflammation in Neurodegenerative Disease-A DoubleEdged Sword, Neuron. 35 (2002) 419-432. https://doi.org/10.1016/S0896-6273(02)00794-8. [10] S. Jinno, F. Fleischer, S. Eckel, V. Schmidt, T. Kosaka, Spatial arrangement of microglia in the mouse hippocampus: A stereological study in comparison with astrocytes, Glia. 55 (2007) 1334-1347. https://doi.org/10.1002/glia.20552.

[11] S. Kondo, S. Kohsaka, S. Okabe, Long-term changes of spine dynamics and microglia after transient peripheral immune response triggered by LPS in vivo, Mol. Brain. 4 (2011) 27. https://doi.org/10.1186/1756-6606-4-27.

[12] R.J. Tynan, S. Naicker, M. Hinwood, E. Nalivaiko, K.M. Buller, D.V. Pow, T.A. Day, F.R. Walker, Chronic stress alters the density and morphology of microglia in a subset of stress-responsive brain regions, Brain. Behav. Immun. 24 (2010) 1058-1068. https://doi.org/10.1016/j.bbi.2010.02.001.

[13] B.B.A. McNaull, S. Todd, B. McGuinness, A.P. Passmore, Inflammation and AntiInflammatory Strategies for Alzheimer's Disease - A Mini-Review, Gerontology. 56 (2010) 3-14. https://doi.org/10.1159/000237873.

[14] J.B. Pitcher, A.M. Riley, S.H. Doeltgen, L. Kurylowicz, J.C. Rothwell, S.M. McAllister, A.E. Smith, A. Clow, D.J. Kennaway, M.C. Ridding, Physiological Evidence Consistent with Reduced Neuroplasticity in Human Adolescents Born Preterm, J. Neurosci. 32 (2012) 16410-16416. https://doi.org/10.1523/JNEUROSCI.3079-12.2012.

[15] M.A. Calcia, D.R. Bonsall, P.S. Bloomfield, S. Selvaraj, T. Barichello, O.D. Howes, Stress and neuroinflammation: a systematic review of the effects of stress on microglia and the implications for mental illness, Psychopharmacology (Berl.). 233 (2016) 1637-1650. https://doi.org/10.1007/s00213-016-4218-9.

[16] W.H. Kaye, J.L. Fudge, M. Paulus, New insights into symptoms and neurocircuit function of anorexia nervosa, Nat. Rev. Neurosci. 10 (2009) 573-584.

https://doi.org/10.1038/nrn2682.

[17] M.G. Stokes, M. Kusunoki, N. Sigala, H. Nili, D. Gaffan, J. Duncan, Dynamic Coding for Cognitive Control in Prefrontal Cortex, Neuron. 78 (2013) 364-375.

https://doi.org/10.1016/j.neuron.2013.01.039.

[18] V. Vilgis, K.L. Gelardi, J.L. Helm, E.E. Forbes, A.E. Hipwell, K. Keenan, A.E. Guyer, Dorsomedial Prefrontal Activity to Sadness Predicts Later Emotion Suppression and

Depression Severity in Adolescent Girls, Child Dev. 89 (2018) 758-772.

https://doi.org/10.1111/cdev.13023.

[19] E.K. Miller, The prefontral cortex and cognitive control, Nat. Rev. Neurosci. 1 (2000) 59-65. https://doi.org/10.1038/35036228.

[20] E.A. Phelps, M.R. Delgado, K.I. Nearing, J.E. LeDoux, Extinction Learning in Humans: Role of the Amygdala and vmPFC, Neuron. 43 (2004) 897-905. https://doi.org/10.1016/j.neuron.2004.08.042.

[21] G. Vanni-Mercier, F. Mauguière, J. Isnard, J.-C. Dreher, The Hippocampus Codes the Uncertainty of Cue-Outcome Associations: An Intracranial Electrophysiological Study in Humans, J. Neurosci. 29 (2009) 5287-5294. https://doi.org/10.1523/JNEUROSCI.529808.2009 .

[22] C. Dahlke, D. Saberi, B. Ott, B. Brand-Saberi, T. Schmitt-John, C. Theiss, Inflammation and neuronal death in the motor cortex of the wobbler mouse, an ALS animal model, J. Neuroinflammation. 12 (2015). https://doi.org/10.1186/s12974-015-0435-0.

[23] J.M. McKlveen, B. Myers, J.N. Flak, J. Bundzikova, M.B. Solomon, K.B. Seroogy, J.P. Herman, Role of Prefrontal Cortex Glucocorticoid Receptors in Stress and Emotion, Biol. Psychiatry. 74 (2013) 672-679. https://doi.org/10.1016/j.biopsych.2013.03.024. 
[24] E.R. de Kloet, M. Joëls, F. Holsboer, Stress and the brain: from adaptation to disease, Nat. Rev. Neurosci. 6 (2005) 463-475. https://doi.org/10.1038/nrn1683.

[25] T. Larrieu, L.M. Hilal, C. Fourrier, V.D. Smedt-Peyrusse, S. N, L. Capuron, S. Layé, Nutritional omega-3 modulates neuronal morphology in the prefrontal cortex along with depression-related behaviour through corticosterone secretion, Transl. Psychiatry. 4 (2014) e437-e437. https://doi.org/10.1038/tp.2014.77.

[26] J.A. Wixey, K.K. Chand, P.B. Colditz, S.T. Bjorkman, Review: Neuroinflammation in intrauterine growth restriction, Placenta. 54 (2017) 117-124.

https://doi.org/10.1016/j.placenta.2016.11.012.

[27] M.B. Alves, D.P. Laureano, R. Dalle Molle, T.D. Machado, A.P. de A. Salvador, P.M. Miguel, D. Lupinsky, C. Dalmaz, P.P. Silveira, Intrauterine growth restriction increases impulsive behavior and is associated with altered dopamine transmission in both medial prefrontal and orbitofrontal cortex in female rats, Physiol. Behav. 204 (2019) 336-346. https://doi.org/10.1016/j.physbeh.2019.03.010.

[28] B. Ajami, J.L. Bennett, C. Krieger, W. Tetzlaff, F.M.V. Rossi, Local self-renewal can sustain CNS microglia maintenance and function throughout adult life, Nat. Neurosci. 10 (2007) 1538-1543. https://doi.org/10.1038/nn2014.

[29] J. Gehrmann, Y. Matsumoto, G.W. Kreutzberg, Microglia: Intrinsic immuneffector cell of the brain, Brain Res. Rev. 20 (1995) 269-287. https://doi.org/10.1016/0165-

0173(94)00015-H.

[30] F. Ginhoux, S. Lim, G. Hoeffel, D. Low, T. Huber, Origin and differentiation of microglia, Front. Cell. Neurosci. 7 (2013). https://doi.org/10.3389/fncel.2013.00045.

[31] U.N. Das, Is obesity an inflammatory condition?, Nutrition. 17 (2001) 953-966. https://doi.org/10.1016/S0899-9007(01)00672-4.

[32] S.D. Bilbo, V. Tsang, Enduring consequences of maternal obesity for brain inflammation and behavior of offspring, FASEB J. 24 (2010) 2104-2115.

https://doi.org/10.1096/fj.09-144014.

[33] G.T. Ray, L.A. Croen, L.A. Habel, Mothers of Children Diagnosed with AttentionDeficit/Hyperactivity Disorder: Health Conditions and Medical Care Utilization in Periods Before and After Birth of the Child, Med. Care. 47 (2009) 105-114. https://doi.org/10.1097/MLR.0b013e31817e18c0.

[34] P.H. Patterson, MATERNAL INFECTION AND IMMUNE INVOLVEMENT IN AUTISM, Trends Mol. Med. 17 (2011) 389-394.

https://doi.org/10.1016/j.molmed.2011.03.001.

[35] L. Schmitz, R. Kuglin, I. Bae-Gartz, R. Janoschek, S. Appel, A. Mesaros, I.

Jakovcevski, C. Vohlen, M. Handwerk, R. Ensenauer, J. Dötsch, E. Hucklenbruch-Rother, Hippocampal insulin resistance links maternal obesity with impaired neuronal plasticity in adult offspring, Psychoneuroendocrinology. 89 (2018) 46-52.

https://doi.org/10.1016/j.psyneuen.2017.12.023.

[36] B. Vuong, G. Odero, S. Rozbacher, M. Stevenson, S.M. Kereliuk, T.J. Pereira, V.W.

Dolinsky, T.M. Kauppinen, Exposure to gestational diabetes mellitus induces

neuroinflammation, derangement of hippocampal neurons, and cognitive changes in rat offspring, J. Neuroinflammation. 14 (2017) 1-13. https://doi.org/10.1186/s12974-017-0859-9.

[37] D. Val-Laillet, Review: Impact of food, gut-brain signals and metabolic status on brain activity in the pig model: 10 years of nutrition research using in vivo brain imaging, Animal. 13 (2019) 2699-2713. https://doi.org/10.1017/S1751731119001745.

[38] E. Roura, S.-J. Koopmans, J.-P. Lallès, I. Le Huerou-Luron, N. de Jager, T.

Schuurman, D. Val-Laillet, Critical review evaluating the pig as a model for human nutritional physiology, Nutr. Res. Rev. 29 (2016) 60-90.

https://doi.org/10.1017/S0954422416000020. 
[39] J. Odle, X. Lin, S.K. Jacobi, S.W. Kim, C.H. Stahl, The Suckling Piglet as an Agrimedical Model for the Study of Pediatric Nutrition and Metabolism, Annu. Rev. Anim. Biosci. 2 (2014) 419-444. https://doi.org/10.1146/annurev-animal-022513-114158.

[40] K. Ferenc, P. Pietrzak, M.M. Godlewski, J. Piwowarski, R. Kilianczyk, P. Guilloteau, R. Zabielski, Intrauterine growth retarded piglet as a model for humans - Studies on the perinatal development of the gut structure and function, Reprod. Biol. 14 (2014) 51-60. https://doi.org/10.1016/j.repbio.2014.01.005.

[41] C.L. Bourgot, S. Ferret- Bernard, E. Apper, B. Taminiau, A. Cahu, L.L. Normand, F. Respondek, I.L. Huërou- Luron, S. Blat, Perinatal short-chain fructooligosaccharides program intestinal microbiota and improve enteroinsular axis function and inflammatory status in high-fat diet-fed adult pigs, FASEB J. 33 (2019) 301-313. https://doi.org/10.1096/fj.201800108R.

[42] D. Val-Laillet, S. Layec, S. Guérin, P. Meurice, C.-H. Malbert, Changes in Brain Activity After a Diet-Induced Obesity, Obesity. 19 (2011) 749-756. https://doi.org/10.1038/oby.2010.292.

[43] P. Sauleau, E. Lapouble, D. Val-Laillet, C.-H. Malbert, The pig model in brain imaging and neurosurgery, Animal. 3 (2009) 1138-1151.

https://doi.org/10.1017/S1751731109004649.

[44] D. Val-Laillet, M. Besson, S. Guérin, N. Coquery, G. Randuineau, A. Kanzari, H. Quesnel, N. Bonhomme, J.E. Bolhuis, B. Kemp, S. Blat, I. Le Huërou-Luron, C. Clouard, A maternal Western diet during gestation and lactation modifies offspring's microbiota activity, blood lipid levels, cognitive responses, and hippocampal neurogenesis in Yucatan pigs, FASEB J. 31 (2017) 2037-2049. https://doi.org/10.1096/fj.201601015R.

[45] Y. Gautier, I. Luneau, N. Coquery, P. Meurice, C.-H. Malbert, S. Guerin, B. Kemp, J.E. Bolhuis, C. Clouard, I.L. Huërou- Luron, S. Blat, D. Val- Laillet, Maternal Western diet during gestation and lactation modifies adult offspring's cognitive and hedonic brain processes, behavior, and metabolism in Yucatan minipigs, FASEB J. 32 (2018) 6478-6794. https://doi.org/10.1096/fj.201701541.

[46] S. Saikali, P. Meurice, P. Sauleau, P.-A. Eliat, P. Bellaud, G. Randuineau, M. Vérin, C.-H. Malbert, A three-dimensional digital segmented and deformable brain atlas of the domestic pig, J. Neurosci. Methods. 192 (2010) 102-109.

https://doi.org/10.1016/j.jneumeth.2010.07.041.

[47] G.W. Kreutzberg, Microglia: a sensor for pathological events in the CNS, Trends Neurosci. 19 (1996) 312-318. https://doi.org/10.1016/0166-2236(96)10049-7.

[48] M. Prinz, J. Priller, Microglia and brain macrophages in the molecular age: from origin to neuropsychiatric disease, Nat. Rev. Neurosci. 15 (2014) 300-312. https://doi.org/10.1038/nrn3722.

[49] H. Kettenmann, U.-K. Hanisch, M. Noda, A. Verkhratsky, Physiology of Microglia, Physiol. Rev. 91 (2011) 461-553. https://doi.org/10.1152/physrev.00011.2010.

[50] C.N. Parkhurst, G. Yang, I. Ninan, J.N. Savas, J.R. Yates, J.J. Lafaille, B.L. Hempstead, D.R. Littman, W.-B. Gan, Microglia promote learning-dependent synapse formation through BDNF, Cell. 155 (2013) 1596-1609.

https://doi.org/10.1016/j.cell.2013.11.030.

[51] L. Nyberg, Cognitive control in the prefrontal cortex: A central or distributed executive?, Scand. J. Psychol. 59 (2018) 62-65. https://doi.org/10.1111/sjop.12409. [52] J. Pearson-Leary, D. Eacret, R. Chen, H. Takano, B. Nicholas, S. Bhatnagar, Inflammation and vascular remodeling in the ventral hippocampus contributes to vulnerability to stress, Transl. Psychiatry. 7 (2017) e1160. https://doi.org/10.1038/tp.2017.122.

[53] M.B. Moser, E.I. Moser, E. Forrest, P. Andersen, R.G. Morris, Spatial learning with a minislab in the dorsal hippocampus, Proc. Natl. Acad. Sci. 92 (1995) 9697-9701. 
https://doi.org/10.1073/pnas.92.21.9697.

[54] K. Brodmann, Brodmann's: Localisation in the Cerebral Cortex, Springer US, 2006. https://www.springer.com/gp/book/9780387269177 (accessed March 2, 2020).

[55] C.A. Heidbreder, H.J. Groenewegen, The medial prefrontal cortex in the rat: evidence for a dorso-ventral distinction based upon functional and anatomical characteristics, Neurosci. Biobehav. Rev. 27 (2003) 555-579. https://doi.org/10.1016/j.neubiorev.2003.09.003.

[56] V. Glykos, M.A. Whittington, F.E.N. LeBeau, Subregional differences in the generation of fast network oscillations in the rat medial prefrontal cortex (mPFC) in vitro, $\mathrm{J}$. Physiol. 593 (2015) 3597-3615. https://doi.org/10.1113/JP270811.

[57] R.P. Kesner, J.C. Churchwell, An analysis of rat prefrontal cortex in mediating executive function, Neurobiol. Learn. Mem. 96 (2011) 417-431.

https://doi.org/10.1016/j.nlm.2011.07.002.

[58] S.C. Cook, C.L. Wellman, Chronic stress alters dendritic morphology in rat medial prefrontal cortex, J. Neurobiol. 60 (2004) 236-248. https://doi.org/10.1002/neu.20025.

[59] L.A.M. Galea, B.S. McEwen, P. Tanapat, T. Deak, R.L. Spencer, F.S. Dhabhar, Sex differences in dendritic atrophy of CA3 pyramidal neurons in response to chronic restraint stress, Neuroscience. 81 (1997) 689-697. https://doi.org/10.1016/S0306-4522(97)00233-9. [60] C. Liston, M.M. Miller, D.S. Goldwater, J.J. Radley, A.B. Rocher, P.R. Hof, J.H. Morrison, B.S. McEwen, Stress-Induced Alterations in Prefrontal Cortical Dendritic Morphology Predict Selective Impairments in Perceptual Attentional Set-Shifting, J. Neurosci. 26 (2006) 7870-7874. https://doi.org/10.1523/JNEUROSCI.1184-06.2006.

[61] R.M. Ransohoff, V.H. Perry, Microglial Physiology: Unique Stimuli, Specialized Responses, Annu. Rev. Immunol. 27 (2009) 119-145.

https://doi.org/10.1146/annurev.immunol.021908.132528.

[62] E. Veniaminova, M. Oplatchikova, L. Bettendorff, E. Kotenkova, A. Lysko, E. Vasilevskaya, A.V. Kalueff, L. Fedulova, A. Umriukhin, K.-P. Lesch, D.C. Anthony, T. Strekalova, Prefrontal cortex inflammation and liver pathologies accompany cognitive and motor deficits following Western diet consumption in non-obese female mice, Life Sci. 241 (2020) 117163. https://doi.org/10.1016/j.lfs.2019.117163.

[63] J.A.S. Gomes, J.F. Silva, A.P. Marçal, G.C. Silva, G.F. Gomes, A.C.P. de Oliveira, V.L. Soares, M.C. Oliveira, A.V.M. Ferreira, D.C. Aguiar, High-refined carbohydrate diet consumption induces neuroinflammation and anxiety-like behavior in mice, J. Nutr. Biochem. 77 (2020) 108317. https://doi.org/10.1016/j.jnutbio.2019.108317. 
Table 1. Composition and nutritional values of the feeds used for sows and piglets. The amount of minor components such as amino-acids and other additives is not reported in this table. Standard diet (SD) sows were fed the gestation and lactation SD feeds. Western diet (WD) sows were fed a high-fat-fructose WD feed during gestation and lactation. All piglets had access to a pre-starter standard diet for piglets (SDP) from postnatal day (PND) 15 to weaning (PND 25), for progressive habituation to solid feed, before being exclusively fed the starter SDP from weaning to the end of the experiment.

\begin{tabular}{|c|c|c|c|c|c|}
\hline & \multicolumn{3}{|c|}{ Sows feeds } & \multicolumn{2}{|c|}{ Piglets feeds } \\
\hline & $\begin{array}{c}\text { Gestation } \\
\text { SD feed }\end{array}$ & $\begin{array}{c}\text { Lactation } \\
\text { SD feed }\end{array}$ & WD feed & $\begin{array}{c}\text { SDP pre- } \\
\text { starter feed }\end{array}$ & $\begin{array}{c}\text { SDP starter } \\
\text { feed }\end{array}$ \\
\hline \multicolumn{6}{|l|}{ Composition (\%) } \\
\hline Wheat & 22 & 25.6 & 6 & & 23.2 \\
\hline Corn & 10 & 12 & & & 25 \\
\hline Barley & 33.9 & 25.68 & 12 & 45.31 & 24.05 \\
\hline Wheat bran & 15 & 10 & 14 & . & \\
\hline Soybean meal & 9 & 18 & 9 & 17.5 & 22.57 \\
\hline Sunflower meal & & & 8 & & \\
\hline Soybean hulls & & & 11 & & \\
\hline Soybean proteins & & & & 2.5 & \\
\hline Corn starch & & & 6.5 & & \\
\hline Sucrose & & & 9.25 & & \\
\hline Fructose & & & 9.25 & & \\
\hline Lard oil & & & 12 & & \\
\hline Cholesterol & & & 1 & & \\
\hline Vegetal oil & 2 & 2 & & 2.3 & 0.45 \\
\hline Molasses & & 3 & & & \\
\hline Beet pulp & 5 & 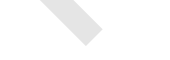 & & & \\
\hline Mild lactoserum & & & & 20 & \\
\hline Fattened milk & & & & 8 & \\
\hline Carbonate calcium & 1.74 & 1.2 & 1.3 & 1.41 & 1.13 \\
\hline Mono-calcic phosphate & & & & 0.8 & 0.97 \\
\hline Bi-calcic phosphate & 0.3 & 1.02 & 0.6 & & \\
\hline Salt & 0.45 & 0.45 & 0.6 & & 0.4 \\
\hline Vitamin complement & 0.5 & 0.5 & 0.5 & 0.5 & 0.5 \\
\hline Crude Protein & 13.32 & 16.45 & 12.18 & 18.99 & 18 \\
\hline Crude fibre & 5.14 & 4.09 & 8 & 2.97 & 3.62 \\
\hline Fat content & 4.28 & 4.21 & 13.45 & 6.74 & 2.79 \\
\hline Dry matter (calculated) & 87.58 & 86.94 & 89.64 & 89.92 & 86.99 \\
\hline Nutritional values & & & & & \\
\hline Net energy, MJ/kg & 9.25 & 9.41 & 14.52 & 10.63 & 9.67 \\
\hline Metabolisable energy, MJ/kg & 13.32 & 12.6 & 18.46 & 13.92 & 12.99 \\
\hline
\end{tabular}


Fig1. A) Representation of the prefrontal cortex (PFC) sections (hematoxylin/eosin coloration). The sites selected in the different PFC subzones, for the counting of Iba1 are Site 3 and Site 6. B) Method of quantification of Iba1 positive microglial cells in the PFC. After selecting two sites (Site3 and Site6) to represent all subzones in the PFC, 10 squares of $1.6 \mathrm{~mm}^{2}$ were selected ( 5 squares for the APFC, 4 for the DLPFC and 1 for the OFC). C) Method of quantification of Iba1 positive microglial cells along the hilus of the hippocampus (HPC) three squares of $1.6 \mathrm{~mm}^{2}$ were used for counting. 
A)

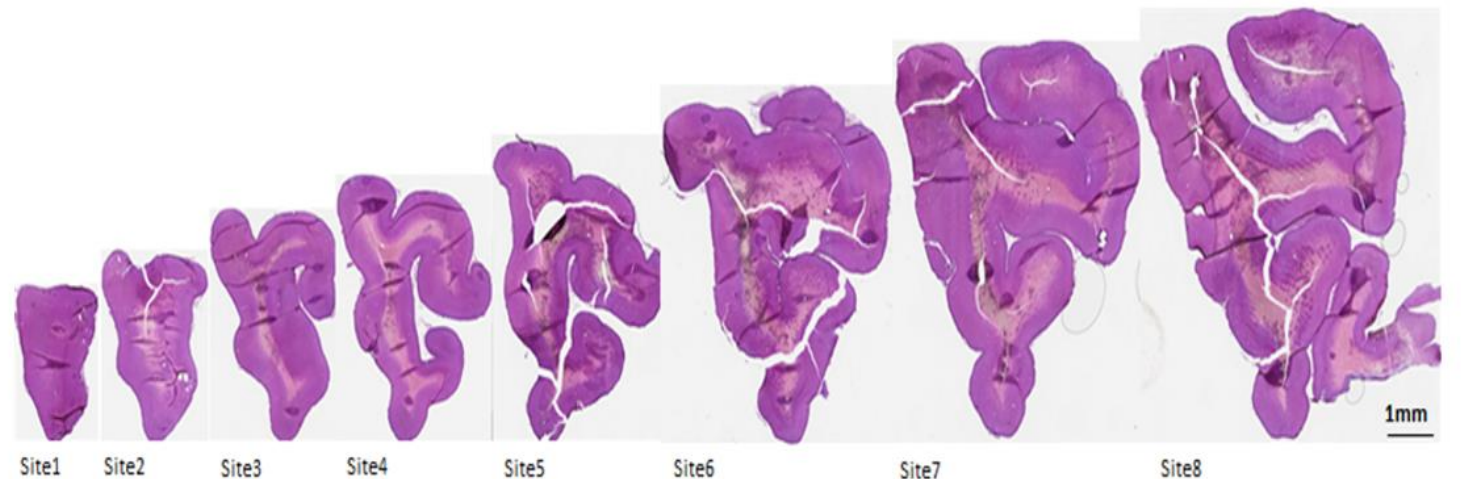

B)

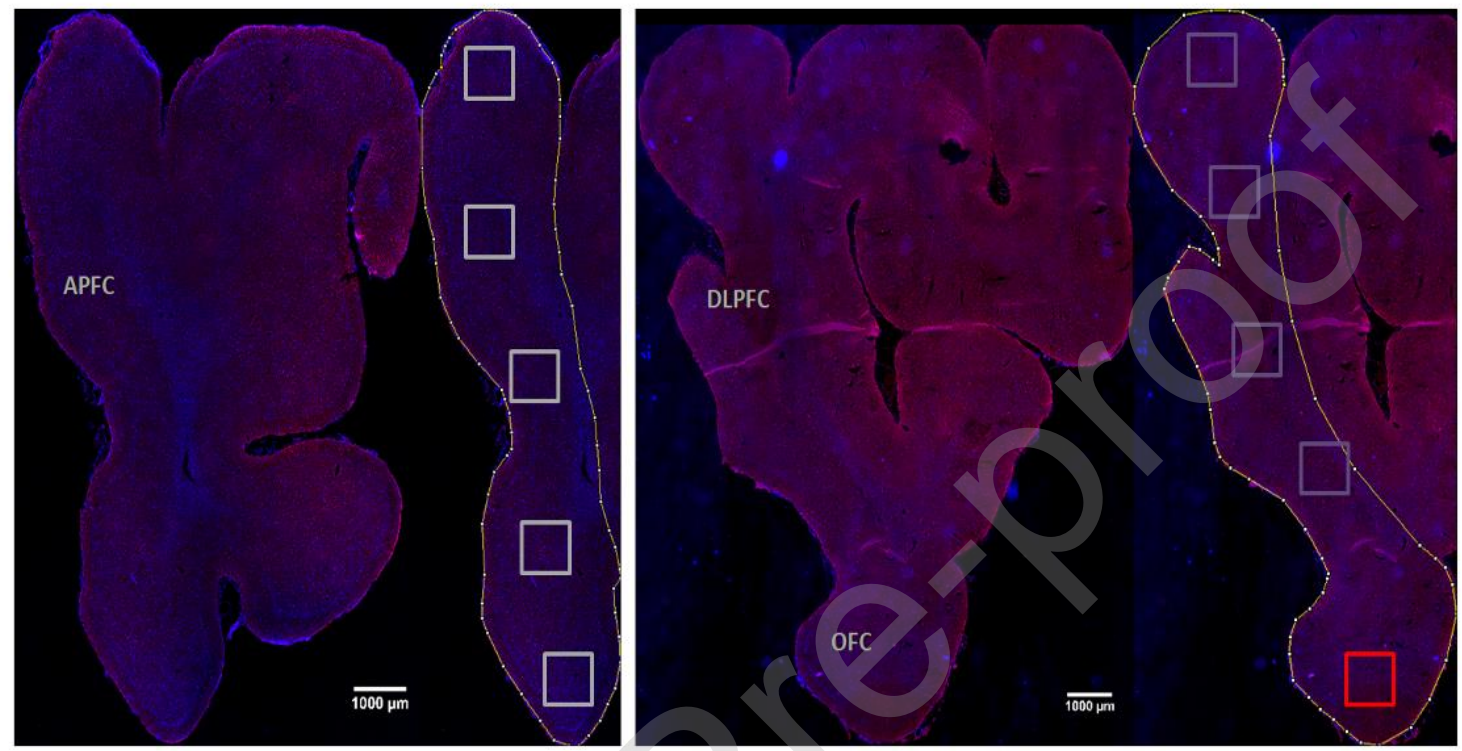

C)

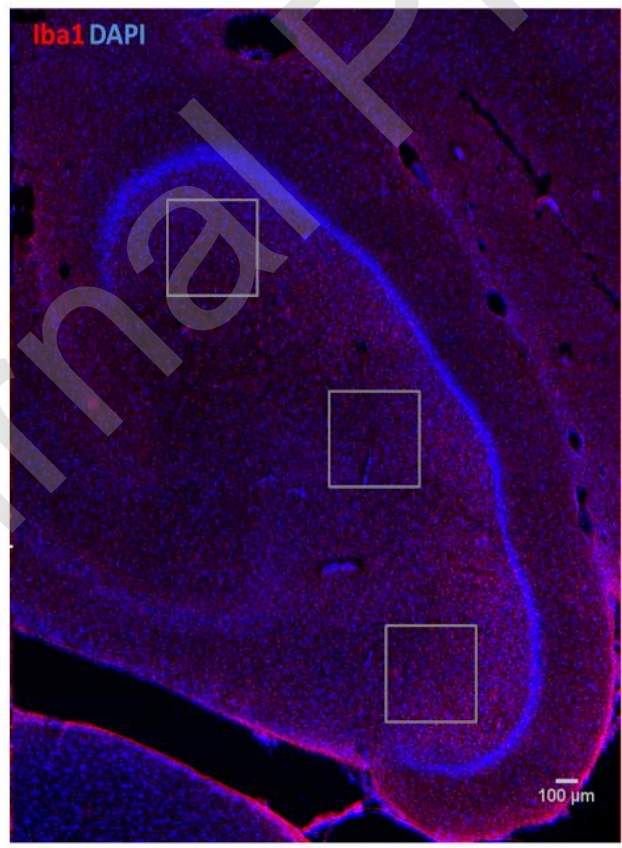

Fig2. Representative images of microglial morphology for cell polarity number evaluation. The different types of microglia are, unipolar with one cytoplasmic 
extension, bi-polar with two cytoplasmic extensions and multi-polar with more than two extensions.

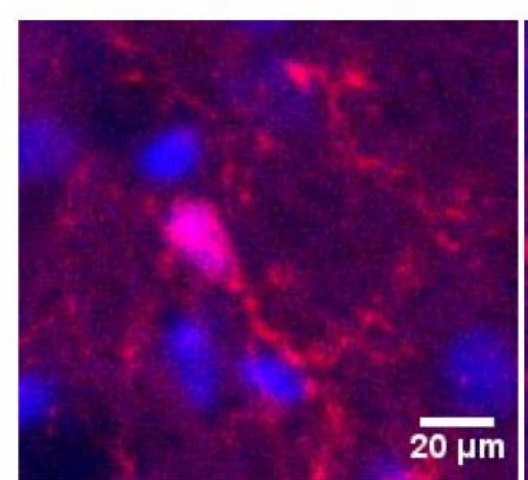

Uni-polar

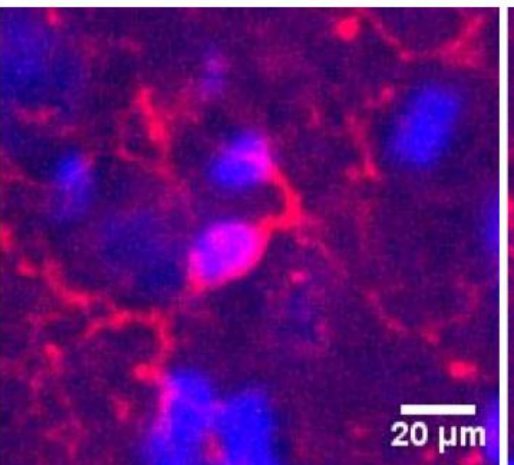

Bi-polar

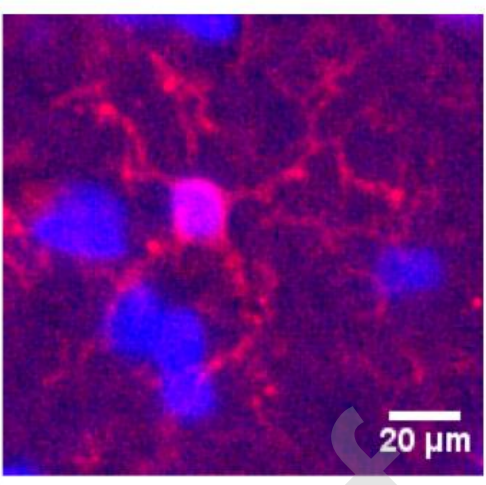

Multi-polar

Fig3. Microglia cell density in the brain (PFC and HPC) of piglets born to sows fed either a standard diet (SD) or a high-fat-fructose western diet (WD) during gestation and lactation. A) Example of the distribution of Iba1 positive microglial cells in the PFC. B) Effects of maternal diet on microglia cell density in the PFC of piglets $(n=16)$. Quantification of Iba1 positive microglia cells in the PFC (SD, n=7 and WD, n=9). Data are expressed as means \pm SEM. ${ }^{* * *} p<0.001$ C) Effects of maternal diet on microglia cell density in the PFC of piglets $(\mathbf{n = 1 5})$. Quantification of Iba1 positive microglia cells in the HPC (SD, $n=7$ and WD, $n=8)$. Data are expressed as means \pm SEM. 
A)

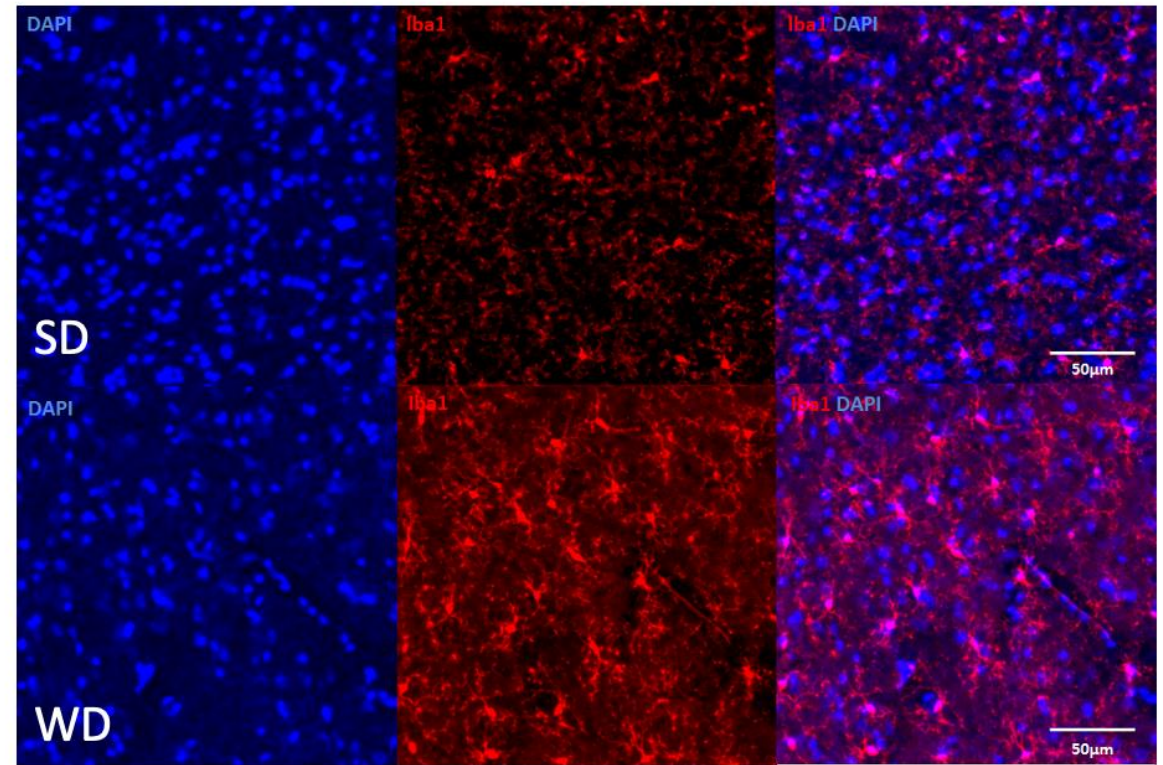

B)

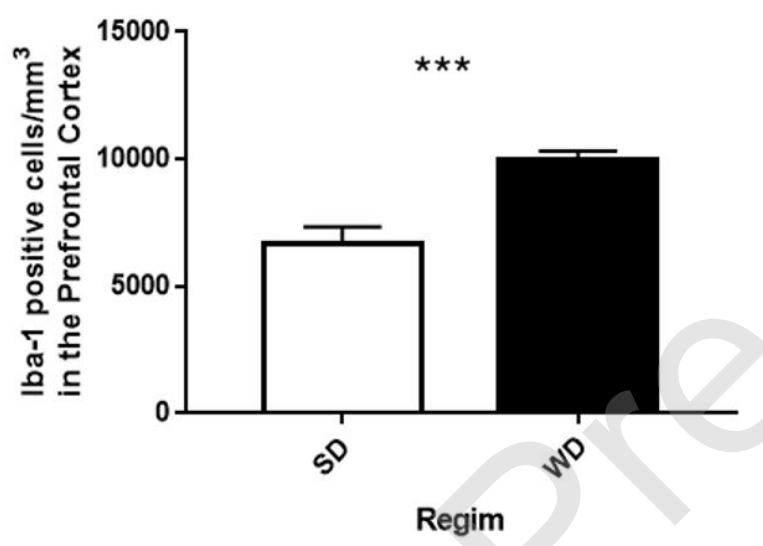

C)

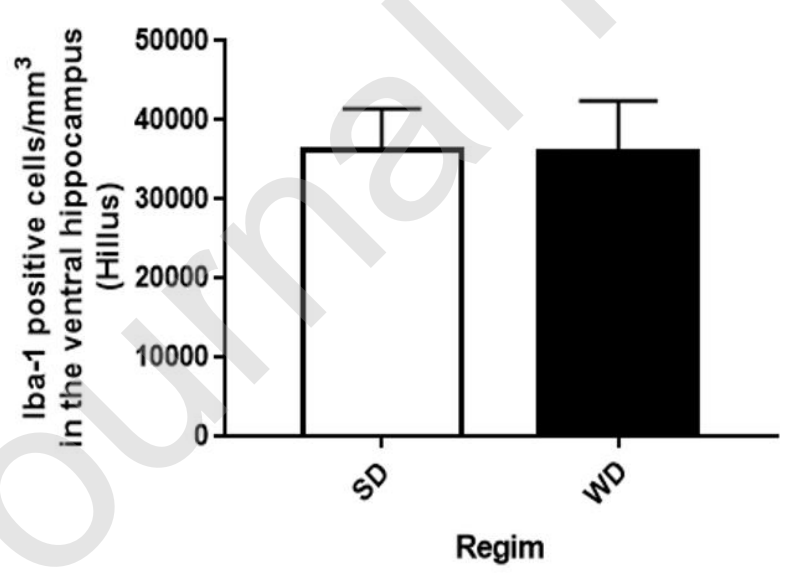

Fig4. Morphology of microglia cells in the PFC and HPC of piglets born to sows fed either a standard diet (SD) or a high-fat-fructose western diet (WD) during gestation and lactation. A) Quantification of the different types of Iba1 positive microglia cells in the different PFC regions, and B) Quantification of the different types of Iba1 positive 
microglia cells in the ventral-HPC. The different types of microglia with the polarity number method are 'Unipolar' with one cytoplasmic extension, 'Bipolar' with two cytoplasmic extensions, and 'Multipolar' with multiple extensions. Data are expressed as means \pm SEM. $* p<0.1, * * p<0.01, * * * p<0.001$, and $* * * * p<0.0001$. 
A)
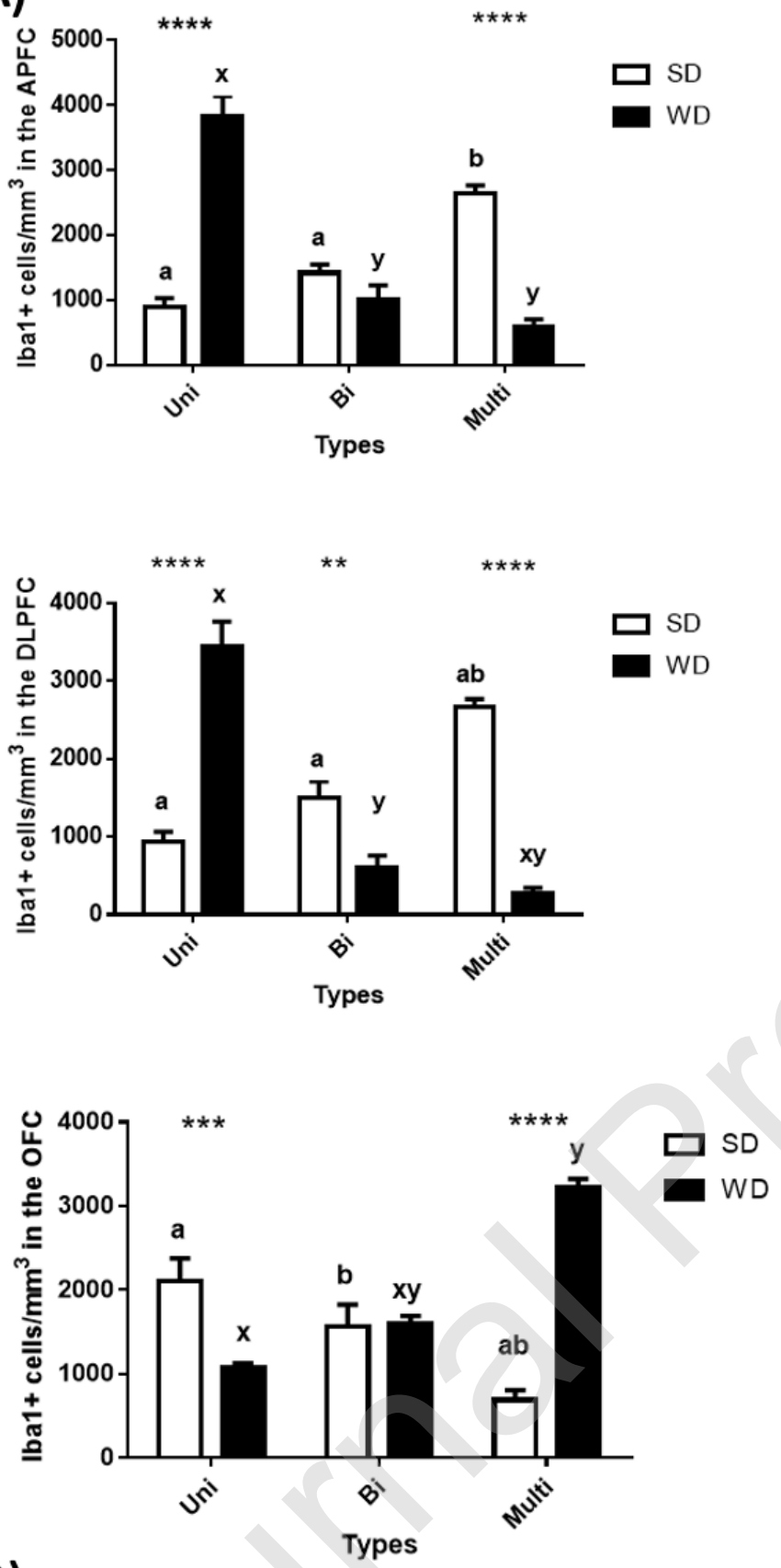

B)

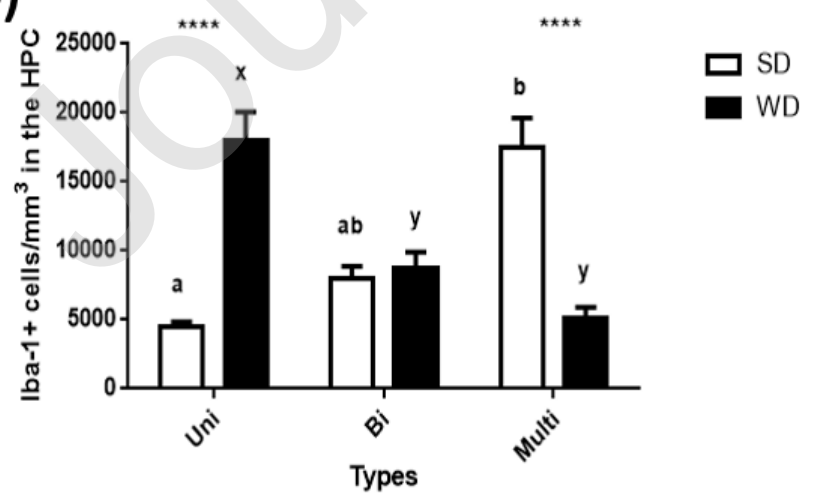

